\title{
Praxis-Induced Myoclonic Seizures are Significantly Associated with Different Self-Perception Trigger Factors
}

Cláudia Cecília da Silva Rêgo ${ }^{1,2}$, Valéria Pereira ${ }^{2}$, Isabella D'Andrea-Meira ${ }^{2}$, Shaylla Vilas Boas Viana ${ }^{1,2}$, Raquel Oliveira ${ }^{1,2}$, Emerson Gasparetto ${ }^{3}$, Jorge Marcondes de Souza ${ }^{4}$ and Soniza Vieira Alves-Leon ${ }^{1,2^{*}}$

${ }^{1}$ Federal University of Rio de Janeiro State, Postgraduation Program of Neurology, Rio de Janeiro, Brazil

${ }^{2}$ Epilepsy Center of University Hospital Clementino Fraga Filho/ Neurology Department, Rio de Janeiro, Brazil

${ }^{3}$ Radiology Department, Hospital Universitário Clementino Fraga Filho, Federal University of Rio de Janeiro Rio de Janeiro, Brazil

${ }^{4}$ Neurosurgery Department, Hospital Universitário Clementino Fraga Filho, Federal University of Rio de Janeiro, Rio de Janeiro, Brazil

*Corresponding author: Soniza Vieira Alves-Leon, Hospital Universitário Clementino Fraga Filho, Universidade Federal do Rio de Janeiro Rua Professor Rodolpho Paulo Rocco 255 - Cidade Universitária, Tel: 55-21-25622712; Fax: 55-21-25622738; Email: sonizavleon@globo.com

Received Date: Mar 16, 2014 Accepted Date: Apr 28, 2014 Published Date: May 5, 2014

Copyright: ( 2014 Rego CCS, et al. This is an open-access article distributed under the terms of the Creative Commons Attribution License, which permits unrestricted use, distribution, and reproduction in any medium, provided the original author and source are credited.

\begin{abstract}
Juvenile myoclonic epilepsy (JME) with praxis-induced seizures consists of a complex group of generalized epileptic syndromes. The aim of the present study was identifying the association between self-perception trigger factors and myoclonic jerks. Twenty-two patients were submitted to a protocol of neuropsychological activation (NPA) during video-electroencephalography (video-EEG) methods for 4-6 hours. A questionnaire was applied regarding patients' self-perception of factors that precipitate of seizures comparing with the video-EEG findings. Stress and sleep deprivation were perceived as triggers by $81.82 \%$, followed by mental concentration (36.36\%) and manual activities (31.82\%). Praxis-induced seizures occurred in $36.4 \%$ of these patients. Self-perception of stress $(81.82 \%)$, flashing lights $(22.73 \%)$ and reading $(13.64 \%)$ as triggering factors was significantly associated with triggering myoclonic seizures during video-EEG. We conclude that main finding of this study is the strong correlation between self-perception of trigger factors and actual trigger factors for seizures.
\end{abstract}

Keywords: Juvenile Myoclonic Epilepsy; Reflex epilepsy; EEG activation; Neuropsychological activation

\section{Introduction}

Juvenile myoclonic epilepsy (JME) is a generalized epilepsy syndrome that corresponds to around $10 \%$ of all cases of epilepsy [1]. Its onset is typically at puberty and it is characterized by the occurrence of myoclonic jerks in all cases, generalized tonic-clonic seizures (GTCS) in $80 \%$ and brief, infrequent absence seizures [1]. Photosensitivity is involved in $30 \%$ of cases [2]. During electroencephalogram (EEG), background activity is normal and the most typical patterns of discharge are the spike-wave and polyspikewave complexes at generalized bursts of 4 to $6 \mathrm{~Hz}$ [3]. Activations such as sleep deprivation are important since they are capable of triggering the onset of epileptic form discharges and myoclonic seizures [4].

Investigation by video-EEG associated with both routine physiological factors and neuropsychological activation has proved to be a robust tool for the diagnosis of JME [5]. Stress and other complex EEG activations, many of which are subjective, have been associated with the triggering of seizures [6,7]. Matsuoka et al. studied 480 patients with different types of epilepsy who were submitted to neuropsychological EEG activation (NPA) [8]. NPA tasks included reading, writing, mental and written arithmetic calculations, spatial construction and putting together a jigsaw puzzle during EEG recording. Those authors recorded epileptic discharges in 38 patients $(7.9 \%)$ and these were accompanied by myoclonic seizures in 15 cases, absence seizures in 8 and simple partial seizures in one. Of the cognitive tasks, mental activities associated with manual tasks were more likely to provoke discharges (68.4\%), showing that NPA is an effective tool with which to evaluate the relationship between cognitive function and epileptic seizures in patients with JME and confirming the susceptibility of this epileptic syndrome to mental activity and praxis. More recently, Guaranhaet al. showed that praxis was the task most likely to provoke discharges $(23.7 \%)$ in 76 patients with JME submitted to neuropsychological activation during video-EEG [9]. Patients who reported that they were anxious had a greater frequency of paroxysms, and traditional activations such as hyperventilation and IPS were more effective than NPA.

The objective of the present study was to investigate patients with JME and ascertain the association between praxis and other NPA tasks in triggering myoclonic seizures and/or discharges in the EEG.

\section{Methods}

Twenty-two patients ( 12 females and 10 males) with JME diagnosed in accordance with the criteria defined by the International League against Epilepsy (ILAE) were included in this study [10]. Patients were recruited at the Epilepsy Center of the ClementinoFragaFilho Teaching Hospital of the Federal University of Rio de Janeiro, Rio de Janeiro, Brazil. The study was approved by the institution's internal review board in accordance with the code of ethics proposed in the Declaration of Helsinki and that of the Brazilian National Health Council (Resolution 196/96 and its revisions). All patients signed an informed consent form.

Patients unable to complete the questionnaire and perform the tasks required in the neuropsychological evaluation, including mentally retarded patients, those with dementia and individuals with 
Citation: Rego CCS, Pereira V, Andrea-Meira I, Viana SVB, Oliveira R, et al., (2014) Praxis-Induced Myoclonic Seizures are Significantly

Page 2 of 6

fewer than four years of formal education, were excluded from the study.

The mean age of disease onset was 13.2 years and the mean duration of epilepsy was 17.5 years (range 1-45 years). All patients were in treatment with antiepileptic drugs (AED), like ValproicAcid, Lamotrigine or Topiramate, none were taking Carbamazepine, Oxcarbazepine or Gabapentin.

A standardized self-perception questionnaire was administered during a personal interview. The questionnaire was based on the model adopted by Antebi\& Bird and Spector et al. and later adapted by Sousa et al. $[7,11,12]$. The objective was to investigate whether these patients were aware of situations that could trigger more seizures. A list of common factors was shown to the patients to assess if they were capable of recognizing any seizure triggers. When these factors were identified, they were compared with the results of activations that occurred during video-EEG. The table $2 \times 2$ and chi-square test was used to analyze associations between self-perception and positive video-EEG findings. Significantly association was considered when $p$ value was $<0.05$.

For this, the patients were monitored in a 192-channel NihonKohden ${ }^{\circ}$ video-EEG device (Tokyo, Japan), model EEG-1200, software Neurofax ${ }^{\oplus}$, version 01-80, CT: $0.3 \backslash \mathrm{s}$, sampling of $200 \mathrm{~Hz}$, impedance < $5 \mathrm{~K} \mathrm{Ohm}$ and filter $70 \mathrm{~Hz}$, Sony camera with image control. The electrode placement system was the complete 10-20 international system. The records were analyzed by two neurologists with experience in this disorder and in evaluating these forms of epilepsy using electroencephalography.

In addition to the routine forms of activation such as eyes open and eyes closed, intermittent photic stimulation (IPS) and hyperventilation, a protocol of NPA tasks was completed during the EEGs. This protocol was based on that defined and published by Matsuoka et al., Mayer \& Wolf and Guaranha et al. $[8,9,13]$. It included reading silently and aloud in Portuguese and in English for 10 minutes each, describing the patient's seizures and the impact of epilepsy on their lives aloud and in writing, performing arithmetical calculations mentally and on paper, spatial construction (consisting of drawing a house and a family), and putting together a jigsaw puzzle. Ten minutes were given for each activity. The video recording of the activations was co-registered with the electroencephalographic tracings.

\section{Results}

When the patients were asked whether they were aware of any situation capable of provoking more seizures, 19 (86.36\%) answered positively. The following responses were obtained: stress and sleep deprivation $(n=18,81.82 \%)$, mental concentration $(n=8 ; 36.36 \%)$, manual work $(n=7 ; 31.82 \%)$, alcohol consumption $(n=6 ; 27.27 \%)$, exposure to flashing lights, making calculations and the menstrual cycle $(n=5 ; 22.73 \%)$, reading $(n=3 ; 13.64 \%)$, playing a musical instrument $(n=2 ; 9.09 \%)$ and listening to music $(n=1 ; 4.55 \%)$.The menstrual cycle was the second most important triggering factor in women, together with praxis involving "mental concentration" and manual work.

Results of the video-EEG tests for each activation showed that 19 $(86.36 \%)$ of the 22 patients had epileptiform discharges during hyperventilation and $18(81.82 \%)$ during IPS. Thirteen patients $(59.09 \%)$ had epileptiform discharges during NPA in the spatial construction segment (Figure 1), 12 (54.55\%) while reading silently and aloud in English, 11 (50\%) while reporting orally on their seizures and when performing written calculations (Figure 2), 9 (40.91\%) when writing about their seizures (Figure 3), 8 (36.36\%) when reading silently and aloud in Portuguese and 6 (27.3\%) while making mental calculations. All 22 patients submitted to NPA and activations such as IPS and hyperventilation were bursts of epileptiform activity during video-EEG monitoring. Of these, 8 (36.4\%) had myoclonic jerks. The same patients who had myoclonic jerks either during special or routine activations were found to have praxis-induced seizures during video-EEG. 
Citation: Rego CCS, Pereira V, Andrea-Meira I, Viana SVB, Oliveira R, et al., (2014) Praxis-Induced Myoclonic Seizures are Significantly Associated with Different Self-Perception Trigger Factors. J Neurol Neurophysiol 5: 204. doi:10.4172/2155-9562-5-1000204

Page 3 of 6

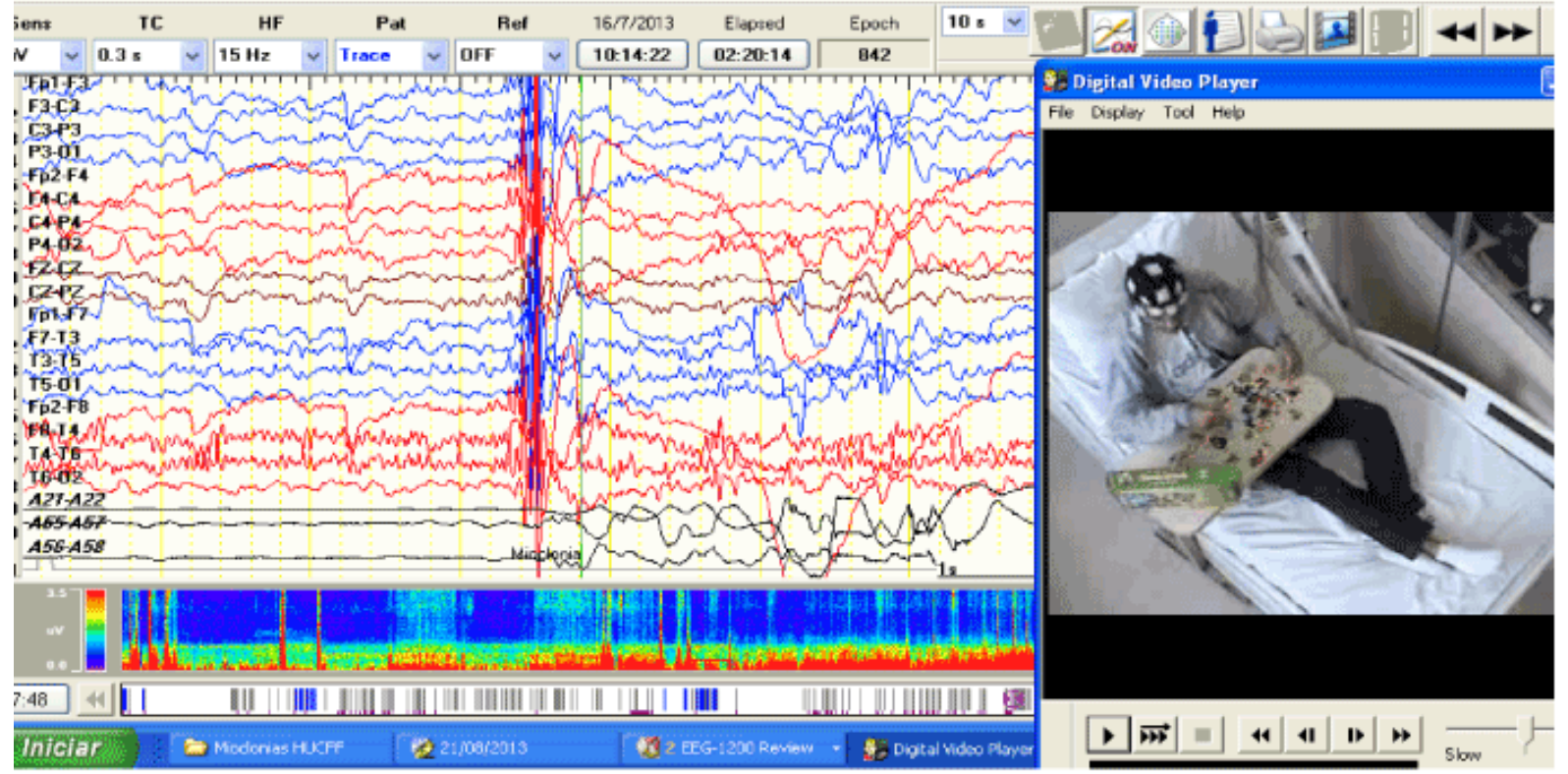

Figure 1. Epileptiform discharges during NPA in the spatial construction segment.

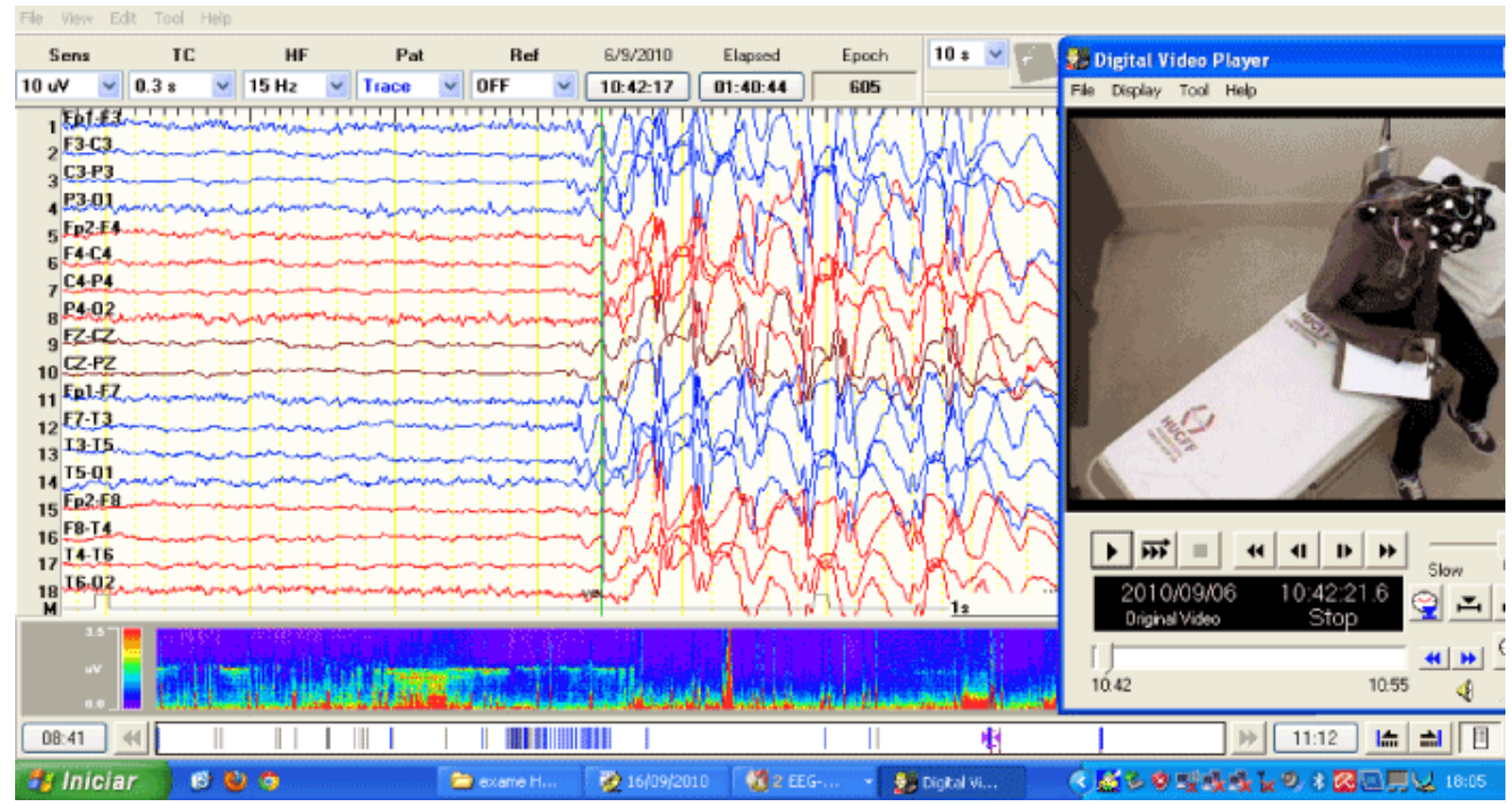

Figure 2. A representative case of a myoclonic seizure when the patient performing written calculations. 
Citation: Rego CCS, Pereira V, Andrea-Meira I, Viana SVB, Oliveira R, et al., (2014) Praxis-Induced Myoclonic Seizures are Significantly

Page 4 of 6

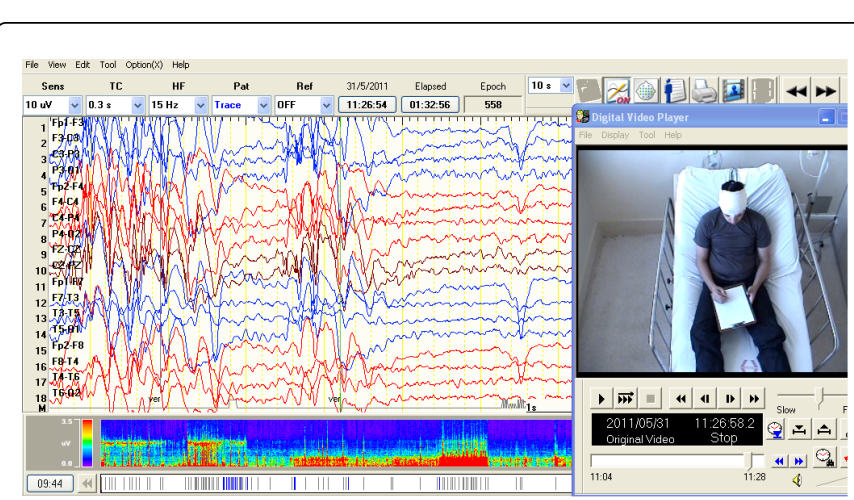

Figure 3: A representative case of two myoclonic seizures when the patient writing about their seizures

Hyperventilation was a trigger of myoclonic jerks in all 8 patients (100\%). It was followed by IPS, reading silently in English and spatial construction in 7 patients (87.5\%), reading aloud in English and making arithmetical calculations on paper in 6 patients (75\%) and reading silently in Portuguese, reading aloud in Portuguese, talking about their seizures, writing about their seizures and making mental arithmetical calculations in 5 patients (62.5\%).

With respect to the results during video-EEG monitoring, the paroxysms most commonly found were the spike-and-slow-wave discharge in 10 patients (45.5\%). Slow-wave was found in 3 patients $(13.6 \%)$, polyspike and spike-and-slow wave in $2(9.1 \%)$, spike-andslow-wave complex in $2(9.1 \%)$, polyspike and spike-and-slow-wave complex in $2(9.1 \%)$, sharp wave and slow wave in $2(9.1 \%)$ and polyspike-slow-wave complex in 1 (4.5\%). The most common minimum frequency found was $4 \mathrm{~Hz}$ in 13 patients (59.1\%), while the maximum was $6 \mathrm{~Hz}$ in 12 patients.

It was found that reading silently and aloud in Portuguese $(\mathrm{p}=0.005)$, speaking $(\mathrm{p}=0.05)$ and writing about the patient's seizures $(\mathrm{p}=0.01)$, making mental $(\mathrm{p}<0.001)$ and written $(\mathrm{p}=0.05)$ calculations and spatial construction $(\mathrm{p}=0.05)$ were significantly associated with triggering seizures (Table 1 ).

The perception of flashing lights triggered seizures was significantly associated with video-EEG records of myoclonic seizures and/or paroxysms during photo-stimulation $(\mathrm{p}<0.001)$, as was reading aloud and silently in English $(\mathrm{p}=0.01)$.On the other hand, no statistically significant correlations werefound between video-EEG and any of the other activations reported by patients in the questionnaire such as concentration, hand movements, calculations, games and writing (Table 1).

\begin{tabular}{|l|l|l|}
\hline $\begin{array}{l}\text { Activations During } \\
\text { Video-Eeg (\%) }\end{array}$ & $\begin{array}{l}\text { Self-Perception Of } \\
\text { Triggering Factors } \\
\text { YesiN0 (\%) }\end{array}$ & P-Value \\
\hline & $\begin{array}{l}\text { Stress } \\
1814(81.814 .2)\end{array}$ & \\
\hline $\begin{array}{l}\text { Reading Silently In } \\
\text { Portuguese } \\
(36.4163 .3)\end{array}$ & 0.005 \\
\hline
\end{tabular}

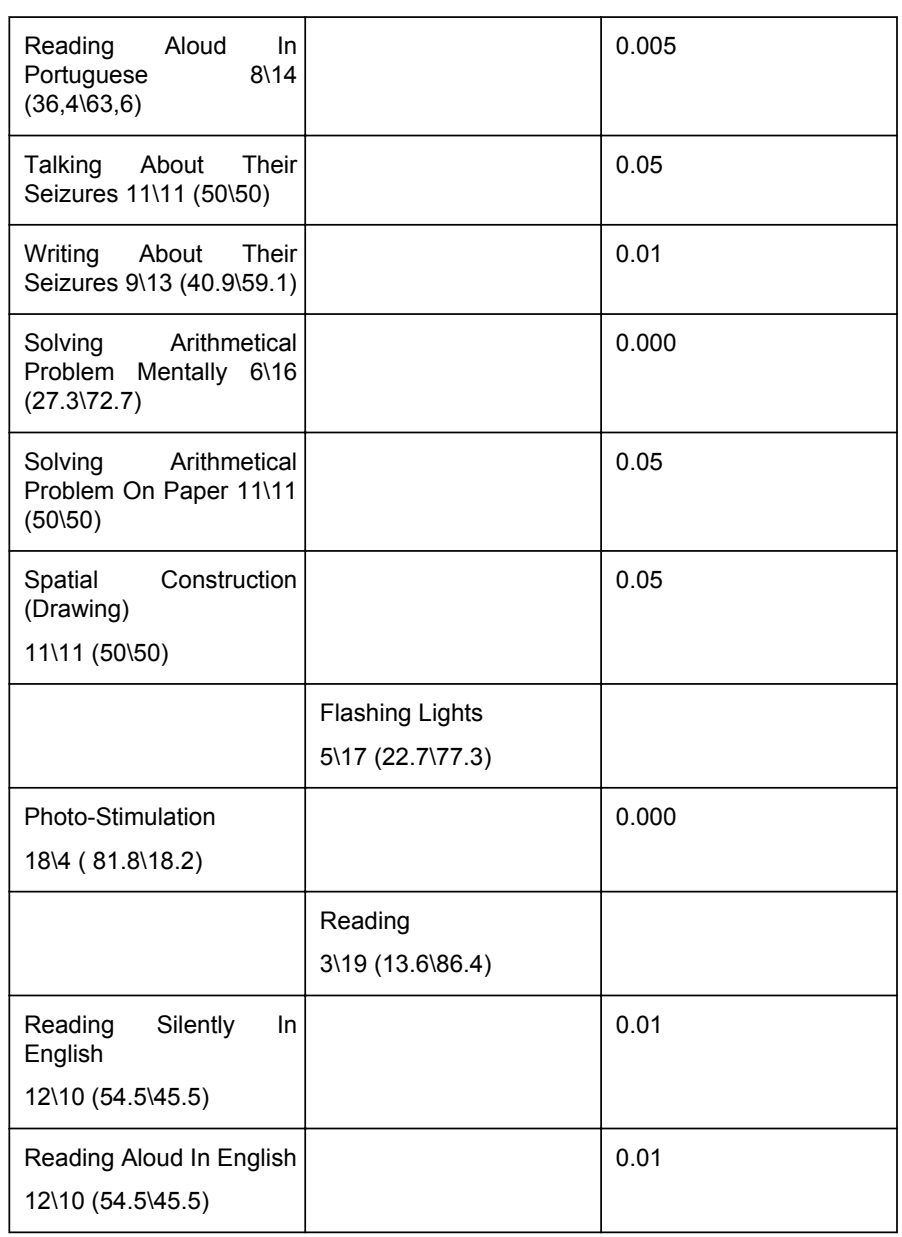

Table 1: Comparison between self-perception of factors capable of triggering myoclonic seizures and confirmation of this end-point during different types of activation associated with stress, flashing lights and reading during video-EEG.

\section{Discussion}

Myoclonic epilepsy with praxis-induced seizures consists of a group of epileptic syndromes that has stimulated investigation on the complex circuits activated by cortical function in idiopathic generalized epilepsies. Therefore, the principal objective of this study was to analyze patients with JME and identify whether activations requiring complex cortical functions such as praxis participate in triggering these seizures.

In the self-perception questionnaire, 19 of the 22 patients (86.36\%) described at least one factor capable of triggering seizures, a percentage similar to that reported by da Silva Sousa et al. (92\%). Beniczky et al. found that cognitive tasks provoke epileptic discharge in $22 \%$ of patients, although, the authors didn't study myoclonic jerks during those discharges [14,15].

In the present series, stress and sleep deprivation were the triggering factors most often reported by patients (both factors being mentioned by 18 patients, $81.82 \%)$. Stress and sleep deprivation were also the most commonly mentioned factors in another study conducted in Brazil (da Silva Sousa et al.), which reported rates of $83 \%$ and $77 \%$, respectively, and in a study carried out in Norway (Kleveland\& Engelson), with rates of $46.5 \%$ and $83.7 \%$, respectively. It 
is interesting to note how stress, mentioned by so many patients as a triggering factor of different morbid conditions and relegated to the background to such an extent by the majority of specialists, was found to have a clear cause and effect relationship with myoclonic jerks. Myoclonia were also most recorded during cognitive tasks by Beniczky et al. [14-16].

Concentration (8 cases, 36.36\%) and manual activities (7 cases, $31.82 \%$ ) were mentioned as factors capable of triggering seizures by around one-third of the present patients. In another four series of patients, concentration was reported by: $6.9 \%$ of patients in India (Murthy et al.), 12\% in Germany (Wolf \& Mayer), 22\% in Saudi Arabia (Panayiotopoulos et al.) and $23 \%$ in Brazil (da Silva Sousa et al.) $[3,17,18]$. The present findings are in agreement with the study conducted in Brazil and with that carried out by Panayiotopoulos et al. ; however, frequencies are higher than those found in the Indian and German populations [3]. These differences may be the result of cultural, genetic or environmental aspects. With respect to manual activities (praxis), similar results (31\%) were found in the German series (Wolf \& Mayer). Lower rates were found, in Japan (12.7\%) (Inoue and Kuboto) and in a previous Brazilian study (20\%) (da Silva Sousa et al.) $[14,18,19]$.

The fourth most commonly mentioned triggering factor in the present series, alcohol consumption (27.27\%) was cited by a higher percentage of patients in Germany (40.4\%) (Janz), Norway (39.5\%) (Kleveland \& Engelsen) and Denmark (51.2\%) (Pedersen \& Petersen) and by a lower percentage of patients in Uruguay (8.6\%) (Castells\&Mendilaharsu) and Brazil (11\%) (da Silva Sousa et al. ) $[14,16,20-22]$. Exposure to flashing lights, the fifth most commonly mentioned triggering factor $(22.73 \%)$ in the present study, was mentioned by $15 \%$ of the patients in a previous Brazilian series (da Silva Sousa et al.) and by 33\% in Uruguay (Castells \& Mendilaharsu), findings that are considered similar to those of the present study $[14,22]$. This factor was more frequently cited in Saudi Arabia (36.8\%) (Panayiotopoulos et al.) and Norway (37.2\%) ( Kleveland \& Engelsen) $[3,16]$. Nevertheless, in another German series (2.1\%) (Janz\& Christian23) and in Japan (10.8\%) (Inoue19) lower rates were reported. Once again, cultural and regional differences may explain these divergences between findings [19,23].

The use of the present questionnaire confirmed the findings of other studies (Matsuoka et al.) (da Silva Sousa et al.) in that patients perceive factors such as stress, sleep deprivation, concentration/ thinking, manual activities (praxis), alcohol consumption and flashing lights as significant triggers of their seizures $[8,14]$. Confirmation of these perceptions during video-EEG provided further data of interest by allowing the results of the self-perception questionnaire on triggering factors to be compared with the recording of both clinical and electroencephalographic phenomena. Indeed, the triggering effect was confirmed in at least one of the activations presented by these 22 patients. Of these, hyperventilation was the factor most likely to trigger seizures, causing discharges in the tracings of 19 patients $(86.36 \%)$, followed by IPS in 18 patients $(81.82 \%)$. These findings confirm the sensitivity of EEG for demonstrating epileptic form activity in patients with JME, as already reported by many authors, including DelgadoEscueta \& Enrile-Bacsal [24], who detected epileptiform activity in $100 \%$ of patients, Janz\& Christian [23], who reported activity in $92 \%$ of cases, Panayiotopoulos et al. in $79 \%$ of cases and Aliberti et al. in $73 \%[3,25]$.

Of the patients in the present study, 8 (36.36\%) developed myoclonic jerks that correlated electrographically with paroxysms in the tracings and praxis-induced seizures. Matsuoka et al. reported that of the 38 patients in their series, myoclonic seizures correlated with paroxysms in the tracing in 15 cases (39.47\%), spatial construction being the activity most likely to provoke myoclonic jerks [8]. Guaranha et al. also reported that of all tasks involving manual activity, spatial construction was the one most likely to provoke myoclonic jerks in their series of 75 patients, accounting for an effect in $15 / 75$ patients tested (20\%), followed by myoclonic jerks triggered by written arithmetical calculations in $12 / 60$ patients (20\%) [9]. The factors identified in the present study as being most likely to trigger myoclonic jerks were: written arithmetical calculations, reading in a foreign language, speaking about the patient's seizures and spatial construction in $70 \%$ of the patients tested, followed by reading in Portuguese, writing about their seizures and mental arithmetic in $30 \%$. Guaranha et al. reported the principal factors as: reading aloud in Portuguese $(7 / 70 ; 10 \%)$, reading aloud in English $(10 / 69 ; 14.5 \%)$, language $(6 / 65 ; 9.2 \%)$, reading silently in Portuguese $(4 / 63 ; 6.3 \%)$, reading silently in English $(5 / 64 ; 7.8 \%)$ and mental calculation $(5 / 66$; 7.6\%) [9].

Regarding interictal EEG, the 22 patients had generalized bilateral discharges, predominantly spike-and-slow-wave (45.5\%) and poly spike-and-slow-wave (9.1\%), with normal background activity. The mean frequency of the discharges found in the present study ranged from $4 \mathrm{~Hz}(59.1 \%)$ to $6 \mathrm{~Hz}(54.5 \%)$. In JME, the spike and poly spikewave discharges are fast and $>3 \mathrm{~Hz}$, generally $4-6 \mathrm{~Hz}$ (Delgado-Escueta \& Enrile-Bacsal), in agreement with the present findings. Spike-wave and polyspike-and-slow-wave discharges are the most prevalent patterns in JME (Guaranha et al., Matsuoka et al.; Janz\& Christian) $[2,8,23,24]$.

Our results can be explained by some knowledge of JME ictogenesis. NPA-induced epileptic discharges are similar to those that develop spontaneously. They consist of diffuse, symmetrical spike and poly spike-wave discharges, but may occur concomitantly with bilateral spike-wave, predominantly over the central region, and may be asymmetrical or not. In JME, a single site or asymmetry is associated with the pathophysiology of myoclonic seizures in the upper limbs (Lancman et al.; Matsuoka, et al.) [26,8]. Aird et al. speculated on whether myoclonic seizures could be caused by pathology in the system projection of the thalamus and central sulcus, in reference to the thalamocortical mechanism involved in absence seizures (Gloor) [27,28]. The clinical semiology of the myoclonic seizures recorded here corroborates the predominance of myoclonus in the upper limbs as a manifestation of NPA-triggered seizures. This motor phenomenon is generally associated with cortical discharges and serves to confirm the paradigm of cortical participation in this epileptic syndrome, as suggested by Vulliemoz et al, i.e. that myoclonic jerks in JME are indeed predominant in the upper limbs and shoulders and are confined to this area in some cases. They may be asymmetrical and may lead to an erroneous diagnosis of focal motor seizure (Lancman et al., Panayiotopoulos et al.) [3,26,29]. Moreover, Vollmaretal showed the increased of functional connective between the motor system and frontoparietal cognitive networks in patients with JME. Our results can be supported by these authors who found that cognitive activation of motor cortex and supplementary motor area in JME explain how cognitive effort can contribute to myoclonic jerks (Vollmar et al) [30].

This study confirms the triggering effect of factors on epileptic discharges and myoclonic seizures at routine examination. An interestingly found was that in some patients, NPA provoked seizures 
exclusively during the performance of mental activities such as reading, writing, drawing and playing. In these cases, praxis took place because the patient was planning an act and performing it manually. Our results, showing that myoclonic jerks were more often confirmed during stress, anxiety, expectation, thinking/concentration and when making decisions, as well as during physiological occurrences such as sleep deprivation, waking and fatigue, suggests that a combination of factors may be common among those patients. Corroborating our results, other authors reported that mental concentration triggered seizures in $22.8 \%$ of patients and stress/expectation in $12.3 \%$ (Panayiotopoulos et al.) [3]. Penryet al. equally reported that seizures in JME were exacerbated by stress (100\%), strong emotions (96\%) and chronic anxiety (70\%) [31]. Indeed, some patients in the present study were unconvinced about the association between myoclonic seizures and triggering factors until they performed the neuropsychological EEG activation tasks. Without this tool, many of the myoclonic seizures induced by mental activity and praxis would have been forgotten or remained unnoticed.

We conclude that main finding of this study is the strong correlation between self-perception of trigger factors and actual trigger factors for seizures. Our results show the importance of self-perception as a relevant an amnestic tool for diagnosing JME, which appears to involve not only the corticothalamic circuit but also other circuits associated with emotional stress. Understanding the interaction of this phenomenon as a whole, including the contribution of stress, will help us understand the pathophysiology of the mechanism of generalized idiopathic epilepsy, principally JME. Finally, this may contribute to the development of a non-pharmacological, cognitive or anti-stress therapeutic intervention for patients with JME.

\section{References}

1. Panayiotopoulos CP, Tahan R, Obeid T (1991) Juvenile myoclonic epilepsy: factors of error involved in the diagnosis and treatment. Epilepsia 32: 672-676.

2. Guaranha MS, Filho GM, Lin K, Guilhoto LM, Caboclo LO, et al. (2011) Prognosis of juvenile myoclonic epilepsy is related to endophenotypes. Seizure 20: 42-48.

3. Panayiotopoulos CP, Obeid T, Tahan AR (1994) Juvenile myoclonic epilepsy: a 5-year prospective study. Epilepsia 35: 285-296.

4. Panayiotopoulos CP, Obeid T, Waheed G (1989) Absences in juvenile myoclonic epilepsy: a clinical and video-electroencephalographic study. Ann Neurol 25: 391-397.

5. Matsuoka H, Takahashi T, Sasaki M, Yoshida S, Numachi Y, et al. (2002) The long-term course of seizure susceptibility in two patients with juvenile myoclonic epilepsy. Seizure 11: 126-130.

6. Andermann F, Zifkin BG, Andermann E (1998) Epilepsy induced by thinking and spatial tasks. In: Zifkin BG, Andermann F, Beaumanoir A, et al. (Eds). Reflex epilepsies and reflex seizures: advances in neurology. Philadelphia: Lippincott-Raven 263-272.

7. Sousa NA, Sousa Pda S, Garzon E, Sakamoto AC, Braga NI, et al. (2005) [EEG recording after sleep deprivation in a series of patients with juvenile myoclonic epilepsy]. Arq Neuropsiquiatr 63: 383-388.

8. Matsuoka H, Takahashi T, Sasaki M, Matsumoto K, Yoshida S, et al. (2000) Neuropsychological EEG activation in patients with epilepsy. Brain 123 : 318-330.

9. Guaranha MS, da Silva Sousa P, de Araújo-Filho GM, Lin K, Guilhoto LM, et al. (2009) Provocative and inhibitory effects of a video-EEG neuropsychologic protocol in juvenile myoclonic epilepsy. Epilepsia 50: 2446-2455.
10. Commission on Classification and Terminology of the International League against Epilepsy. Proposal for revised classification of epilepsies syndromes. Epilepsia 30: 389-399.

11. Antebi D, Bird J (1993) The facilitation and evocation of seizures. A questionnaire study of awareness and control. Br J Psychiatry 162: 759-764.

12. Spector S, Cull C, Goldstein LH (2000) Seizure precipitants and perceived self-control of seizures in adults with poorly-controlled epilepsy. Epilepsy Res 38: 207-216.

13. Mayer T, Wolf P (2004) Perioral reflex myoclonias in reading epilepsy and juvenile myoclonic epilepsy. In: Wolf P, Inoue Y,Zifkin B. (Eds) Reflex epilepsies: progress in understanding. London: John Libbey 57-70.

14. da Silva Sousa P, Lin K, Garzon E, Sakamoto AC, Yacubian EM (2005) Self-perception of factors that precipitate or inhibit seizures in juvenile myoclonic epilepsy. Seizure 14: 340-346.

15. Beniczky S, Guaranha MS, Conradsen I, Singh MB, Rutar V, et al. (2012) Modulation of epileptiform EEG discharges in juvenile myoclonic epilepsy: an investigation of reflex epileptic traits. Epilepsia 53: 832-839.

16. Kleveland G, Engelsen BA (1998) Juvenile myoclonic epilepsy: clinical characteristics, treatment and prognosis in a Norwegian population of patients. Seizure 7: 31-38.

17. Murthy JM, Rao CM, Meena AK (1998) Clinical observations of juvenile myoclonic epilepsy in 131 patients: a study in South India. Seizure 7: 43-47.

18. Wolf P, Mayer T (2000) Juvenile myoclonic epilepsy: a syndrome challenging syndromics concepts. In: Schmitz B, Sander T. (Eds). Juvenile myoclonic epilepsy: The Janz syndrome. Petersfield: Wrightson Biomedical Publishing Ltd 33-40.

19. Inoue Y, Kubota H (2000) Juvenile myoclonic epilepsy with praxisinduced seizures. In: Schmitz B, Sander T. Juvenile myoclonic epilepsy : the Janz syndrome. Petersfield: Wrightson 73-81.

20. Janz D (1985) Epilepsy with impulsive petit mal (juvenile myoclonic epilepsy). Acta Neurol Scand 72: 449-459.

21. Pedersen SB, Petersen KA (1998) Juvenile myoclonic epilepsy: clinical and EEG features. Acta Neurol Scand 97: 160-163.

22. Castells C and Mendilaharsu C (1958) La epilepsia mioclónica bilateral y consciente (Considerações clínicas y fisiopatológicas). Acta NeurolLatinoam 4: 23-48.

23. Janz D, Christian W (1957) Impulsive-petit mal. Deutsche Zeitschrift Nervenheilkunde 176 : 346-386.

24. Delgado-Escueta AV, Enrile-Bacsal F (1984) Juvenile myoclonic epilepsy of Janz. Neurology 34: 285-294.

25. Aliberti V, Grünewald RA, Panayiotopoulos CP, Chroni E (1994) Focal electroencephalographic abnormalities in juvenile myoclonic epilepsy. Epilepsia 35: 297-301.

26. Lancman ME, Asconapé JJ, Penry JK (1994) Clinical and EEG asymmetries in juvenile myoclonic epilepsy. Epilepsia 35: 302-306.

27. Aird RB, Masland RL, Woodbury DM. The epilepsies: a critical review. New York: Raven Press, 1984.

28. Gloor P (1979) Generalized epilepsy with spike-and-wave discharge: a reinterpretation of its electrographic and clinical manifestations. The 1977 William G. Lennox Lecture, American Epilepsy Society. Epilepsia 20: 571-588.

29. Vulliemoz S, Vollmar C, Koepp MJ, Yogarajah M, O'Muircheartaigh J, et al. (2011) Connectivity of the supplementary motor area in juvenile myoclonic epilepsy and frontal lobe epilepsy. Epilepsia 52: 507-514.

30. Vollmar C, O'Muircheartaigh J, Barker GJ, Symms MR, Thompson P, et al. (2011) Motor system hyperconnectivity in juvenile myoclonic epilepsy: a cognitive functional magnetic resonance imaging study. Brain 134: 1710-1719.

31. Penry JK, Dean JC, Riela AR (1989) Juvenile myoclonic epilepsy: longterm response to therapy. Epilepsia 30 Suppl 4: S19-23. 